\title{
Diaper Rash: Frequency, Causes and Type of Inflammation among Under Five Years Old Libyan Pediatric Patients
}

\section{Elfaituri SS* \\ Dermatology department, Benghazi University, Libya}

*Corresponding author: Safa Suleman Elfaituri, Dermatology department, medical faculty, Benghazi university, Elfoihat, 2026 Benghazi, Libya, Tel: 00218925496566;

E-mail: selfaitoury@yahoo.co.uk

\section{Research Article \\ Volume 1 Issue 1}

Received Date: November 08, 2016

Published Date: November 28, 2016

DOI: $10.23880 /$ cdoaj16000102

\section{Abstract}

Diaper rash (DR) is a non-specific term used to describe any inflammatory skin reaction in the diaper area. It is encompassing a range of dermatoses of various causes. Our aim was to study DR among under 5 years Libyan pediatric dermatology patients. A total number of 618 pediatric patients were studied over a period of 1 year. Examination of napkin area was done when the presenting problem was related to diaper area and relevant investigations were performed. DR was seen in $16 \%$ with $70 \%$ of the cases were infants. DR that caused by wearing of diapers was the most frequent (47\%); contact dermatitis (40\%), intertrigon (5\%) and miliaria (2\%). DR that occur irrespective of diaper use constituted $27 \%$, it included infections (13\%), zinc deficiency and Job syndrome (3\%). Involvement of napkin area as a part of erythroderma constituted 8\%. Rashes that appears elsewhere but exaggerated in the napkin area were seen in 26\%; atopic dermatitis (14\%), seborrhoeic dermatitis (8\%) and psoriatic DR (4\%).One year diaper dermatitis study in the Libyan pediatric population has led to a better understanding of the frequency of diaper rash among pediatric dermatology patients and the variable causes. Awareness of the differential diagnosis of diaper rash is important and allows doctors and parents to better assess and manage the symptoms.

Keywords: Diaper rash; Libyan pediatric; Dermatoses

\section{Introduction}

Diaper rash (DR) is a non-specific broad term used to encompass a wide range of inflammatory skin reaction of various causes that occurs in the area covered by the diaper. It can be classified into three main categories: DR that occurs irrespective of diaper use including acrodermatitis enteropathica, Langerhans cell histiocytosis, bullous impetigo, scabies, and HIV,DR that appear elsewhere but can be exaggerated in napkin area due to the irritating effects of wearing a diaper including atopic dermatitis, seborrheic dermatitis, and psoriasis, and DR that are caused by wearing of diapers including irritant and allergic contact dermatitis, miliaria, intertrigo and candidal diaper dermatitis [1,2]. 


\section{Aim of the Study}

Our objective was to study napkin dermatitis in under five year's Libyan pediatric population; to determine the frequency of DR among pediatric dermatology patients, and to identify variable causes of the DR.

\section{Methods}

A prospective study carried out in Jumhoria hospital, Benghazi, Libya for a period of 1 year from January 2012. It involved pediatric patients less than five years of age attending dermatology clinics. A detailed history was recorded and complete dermatological examination including napkin area was done. Relevant investigations including skin biopsy were performed.

\section{Results}

A total of 618 pediatric patients were studied; all were less than 5 years Libyan patients.

A male preponderance (53\%) was observed, infants constituted the largest group within the patient population (52\%).

Diaper rash was seen in 100 cases $(16 \%)$ of our pediatric patients; with most of the cases were infants (70\%). DR was a presenting complains in $50 \%$ of the neonates' patients, $22 \%$ of infants and $6.7 \%$ of preschool children (Figure 1).

In the analysis of diagnoses by categories (Figure 2), DR that caused by wearing of diapers (Figures 3 a-d) was the most frequent (47\%), it included contact dermatitis; irritant or allergic (40\%), intertrigon (5\%) and miliaria (2\%). Secondary candidal DR was diagnosed in $17 \%$. Majority of contact DR was involving convexity of genitalia and buttocks (30\%), 6\% was perianal dermatitis and $4 \%$ was marginal dermatitis.

DR that occur irrespective of diaper use (Figures 4 a-f) were seen in 27\%; it included hand, foot and mouth disease involving diaper area (4\%), zinc deficiency and Job syndrome (3\%), Tinea corporis, ecthyma and bullous impetigo (2\%) whereas, nodular scabies, herpes simplex, and pityriasis rosea were seen only in single cases.

Involvement of napkin area as a part of erythroderma constituted $8 \%$ of DR; scarlet fever (4\%), colloidon baby (2\%), hairlquin baby and staphylococcal scalded skin syndrome $(1 \%)$.

Dermatoses that appear elsewhere but exaggerated in the napkin area (Figures 5 a-c) were seen in 26\%; they included atopic dermatitis (14\%), Seborrhoeic dermatitis (8\%) and psoriatic DR (4\%).

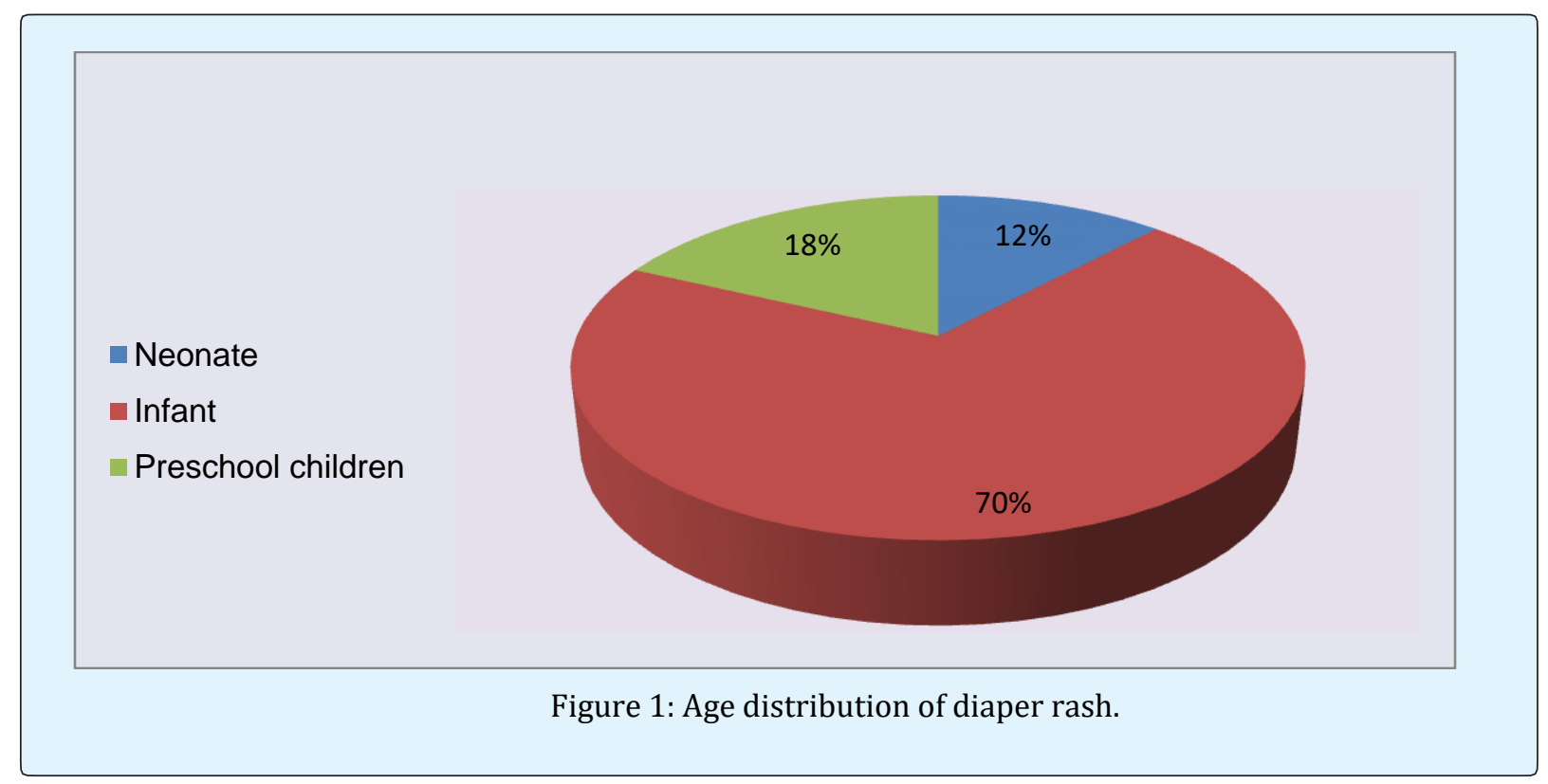

Elfaituri SS, et al. Diaper Rash: Frequency, Causes and Type of Inflammation among Under Five Years Old Libyan Pediatric Patients. Clin Dermatol J 2016, 1(1): 000102. 


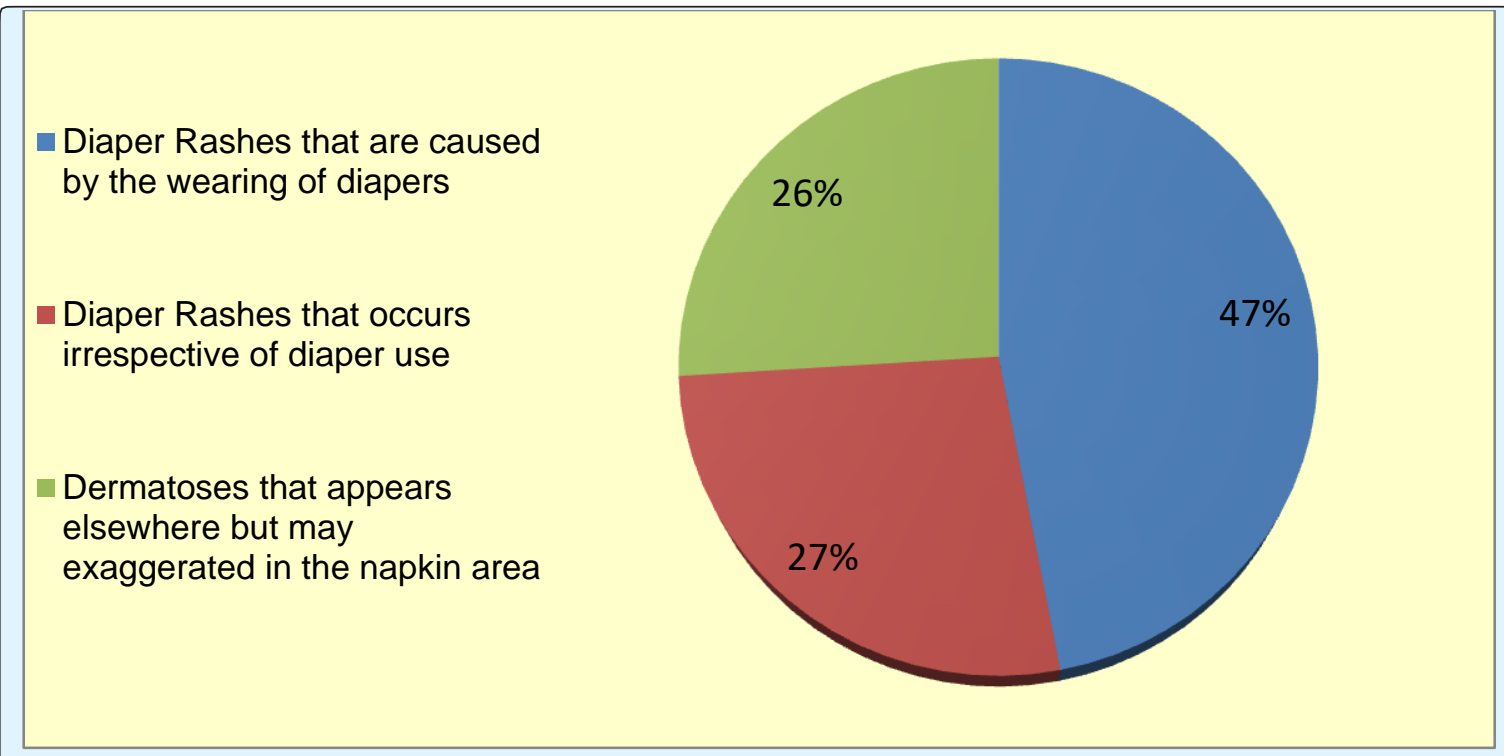

Figure 2: Categories of diaper rash.

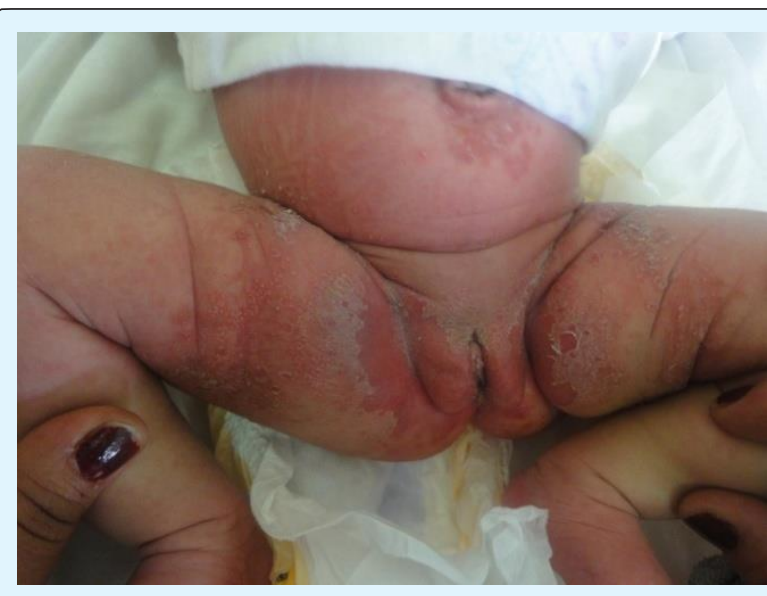

Figure 3a: Diaper rash with 2ry candidiasis.

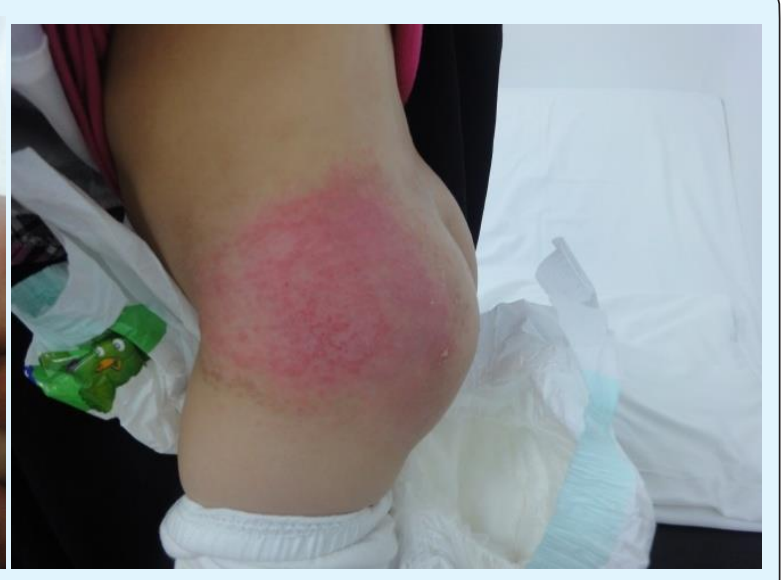

Figure 3b: Marginal dermatitis.

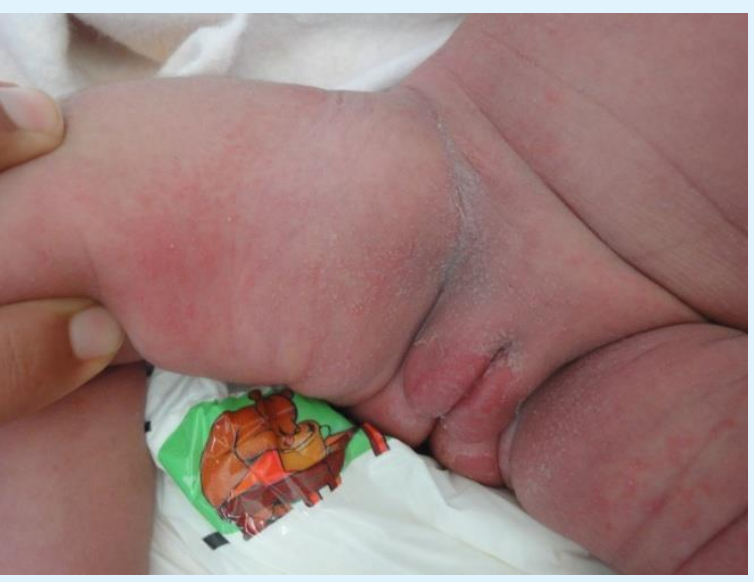

Figure 3d: Miliaria in diaper area.

Figure 3c: Intertrigon. 


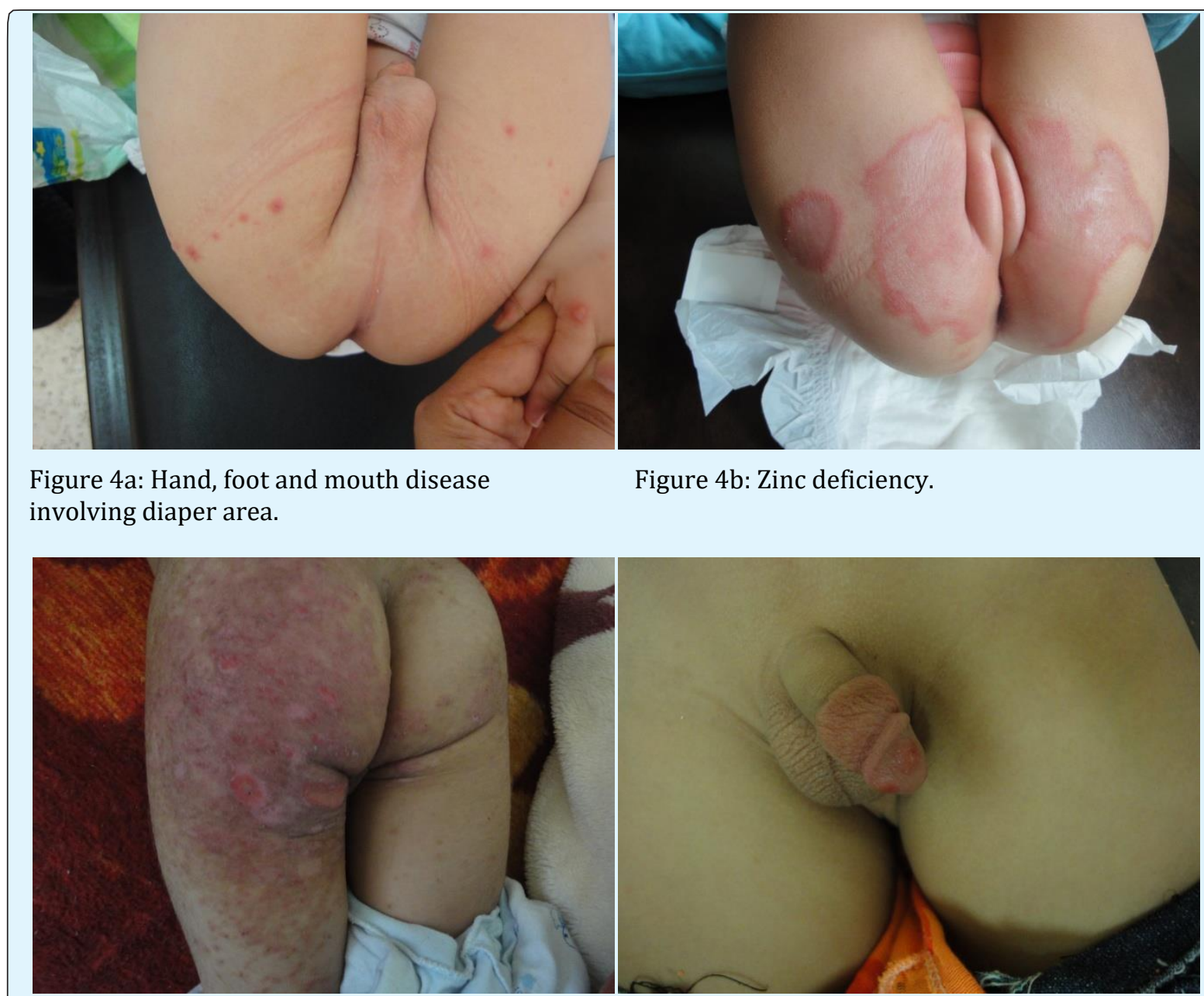

Figure 4c: Chronic eczema with ulcers in Job syndrome.

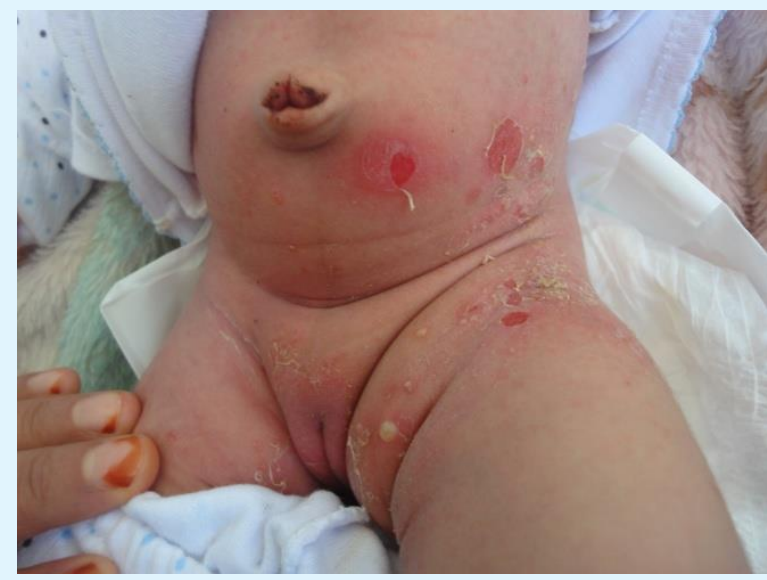

Figure 4e: Bullous impetigo.
Figure 4d: Nodular scabies.

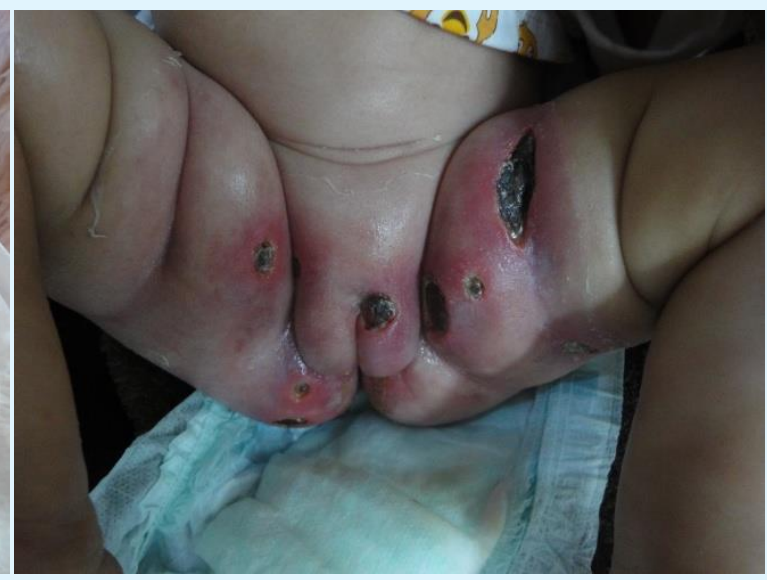

Figure 4f: Ecthymagangreenosum. 


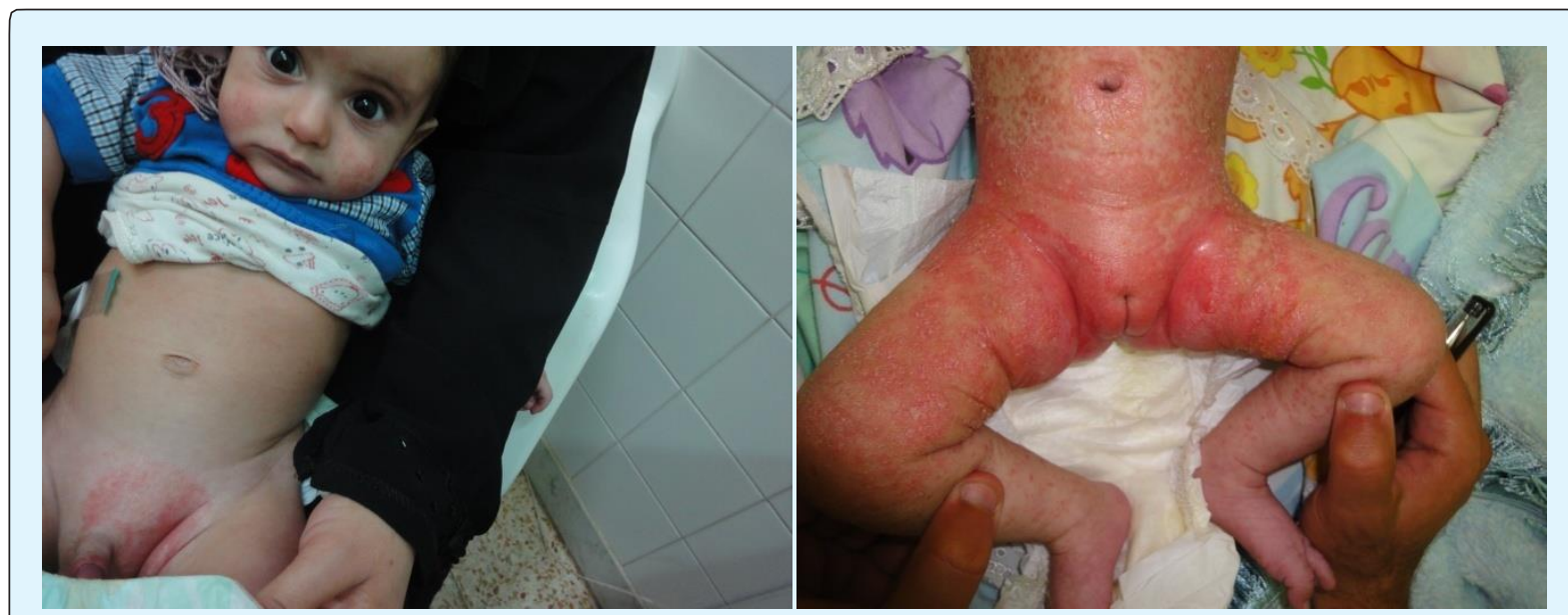

Figure 5a: Atopic dermatitis involving napkin area. Figure 5b: Seborrhoeic dermatitis.

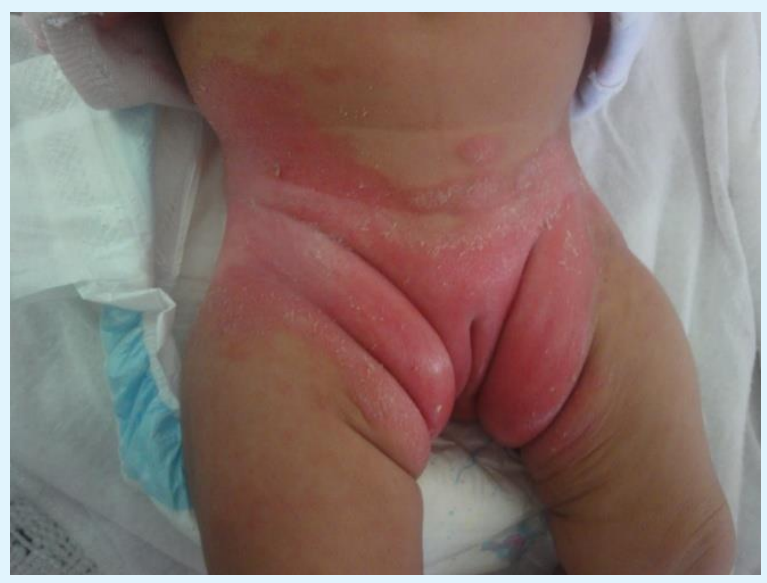

Figure 5c: Psoriatic diaper rash.

\section{Discussion}

Diaper rash is a common problem in infants and young children. The incidence and causes varies greatly between the reports. Our frequency $(16 \%)$ is higher than in Kuwait (4\% ) and Nigeria(7\%) but near to Great Britain data $(20 \%)$ in 1970 s $[2,3]$.

DR caused by wearing of diapers are truly DR because they can be cured by a change in diapering practices. Contact dermatitis (40\%) was mainly involving convexities where the skin is in greatest contact with the diaper. Perianal dermatitis is related to the irritant substances found in stool whereas marginal dermatitis could be due to friction from diaper margin or allergy from marginal dyes or nylon. Secondary candidiasis occurs once the skin is compromised, in this study clinical criteria as satellite papules and pustules with folds involvement were used for diagnosis. Miliaria may occur in any occluded area including diaper area due to over hydration of stratum corneum and occlusion of eccrine sweat glands. Intertrigon of nappy area folds occurs due to wetness, friction and maceration, commonly in obese infants $[4,5]$.

Regarding DR that occur irrespective of diaper use; acrodermatitis enteropathica patients had DR associated with diarrhea and facial periorificial dermatitis. Hyper IgE syndrome patients exhibited chronic refractory DR complicated by staphylococcal and candidal infections.

Staphylococci, streptococci and fecal bacteria can cause infections in diaper area; humidity and warmth are predisposing factors. In this study napkin area was involved by various infections. Coxsackie virus infection with blisters in the hand, foot, and mouth had involved diaper area on the buttocks. Presence of herpes in napkin area should arouse suspicion of sexual abuse. It could be 
due to contact with whitlow in mother's hand. Tinea corporis at napkin site is most likely due to anthropophilic infection, treatment with topical steroid may cause tinea incognito. Scabies is a parasitic infestation that may cause DR, nodular scabies is a variant can persist for several months in genitalia despite successful treatment. Infants with atopic dermatitis are prone to DR because of their sensitivity to irritants, allergens and infections. Seborrheic dermatitis frequently cause DR with fold involvement without satellite lesions [1-3]. Diaper wearing may aggravate these endogenous eczema. Initial lesions of psoriasis in infancy are most often in diaper area as friction and irritation may initiate psoriasis in genetically predisposed individuals [6].

\section{Conclusion}

DR is a common problem among Libyan pediatric patients with nearly half of cases directly or indirectly caused by the wearing of diapers and related to diaper environment. The nappy area can be involved by a variety of dermatoses, awareness of cutaneous mimickers of irritant DR along with careful skin examination as well as detailed history and sometimes histological examination may be needed for accurate diagnosis.

\section{References}

1. Fernandes JD, Machado MCR, Oliveira ZNP (2009) Clinical presentation and treatment of diaper dermatitis - Parte II. An Bras Dermatol 84(1): 47-54.

2. Serdaroğlu S, stünbaş TK (2010) Diaper dermatitis (review). J Turk Acad Dermatol 4(4): 04401r.

3. Kazzi AA, Dib R (2006) Pediatrics, Diaper Rash.

4. KellenPE (1990) Diaper dermatitis: differential diagnosis and management. Can Fam Physician 36: 1569-1572.

5. Rasmussen JE (1987) Classification of diaper dermatitis: an overview. Pediatrician 14(1): 6-10.

6. Saha A, Batra P (2006) Neonatal psoriasis. Indian Pediatrics 43(17): 182-183. 